\title{
IMMUNE RESPONSE CAPABILITY OF `SHUVRA’ CHICKEN
}

\author{
M. S. Sabrin, S. Saha* and M.M. Amin \\ Department of Microbiology and Hygiene, Bangladesh Agricultural University, Mymensingh 2202, Bangladesh
}

\begin{abstract}
The study was carried out to determine the humoral immune response to Newcastle Disease Virus (NDV) and Bangladesh Agricultural University Fowl cholera (BAUFC) vaccines in Shuvra chicken, a newly develop chicken strain by BLRI (Bangladesh Livestock Research Institute). Ten Shuvra chickens were vaccinated with Baby Chick Ranikhet Disease Vaccine (BCRDV) at day 7 through intra ocular route (i/o) and with Ranikhet Disease Vaccine (RDV) at day 35 through intramuscular (i/m) route. Vaccine induced serum Haemagglunination Inhibition (HI) antibodies were measured by HI test. Two weeks after final immunization all vaccinated and control Shuvra chickens were challenged with virulent field isolates of NDV where all the vaccinated birds survived without showing any typical signs of NDV during the period of ten days observation period and all the control chickens died. Another 10 Shuvra were vaccinated twice with BAUFC vaccine through intramuscular route at day 42 and 70, and 10 Shuvra chickens were kept as unvaccinated control. This vaccine also induced significantly higher level of antibody titre as determined by Passive Haemagglutination (PHA) test. Vaccinated chickens showed significantly higher survival $(80 \%)$ following challenge with virulent fowl cholera isolate and all the control birds died within 10 days of observation period.
\end{abstract}

Key words: Shuvra, chick, vaccine, Passive Haemaglutination assay (PHA), antibody titre, fowl cholera

\section{INTRODUCTION}

In Bangladesh chicken contributes $51 \%$ of the total meat production (FAO, 1999; BBS, 2009). Per capita annual consumption of meat in this country is $5.9 \mathrm{~kg}$ which is very low and only $7.98 \%$ of the universal standard (MoFL, 2006). The annual per capita egg consumption is only 23 although it should be 100 from a nutritional point of view. The predominant poultry breed in Bangladesh is local, kept in scavenging system. The productivity of a local hen is 40-60 eggs per year (Saleque and Mustafa, 1996). To develop a high yield layer strain of chicken, Bangladesh Livestock Research Institute (BLRI) has carried out a long strenuous research along with Japan International Cooperation Agency (JICA). Shuvra is the product of such research which is claimed to be very adaptable to rural condition. Shuvra start laying eggs within 19 weeks and an annual laying capacity of eggs is around 280 to 295 (BLRI).

Among infectious diseases of backyard poultry, Newcastle disease (ND) and fowl cholera (FC) are highly endemic (Siddique et al., 1997; Samad, 2000) in Bangladesh. Although vaccination is being practiced to control NDV in Bangladesh but outbreaks of NDV infection has been reported by several investigators (Sarkar et. al., 2012, Rahman et. al., 2004).

Fowl Cholera (FC) is also a disease of economic importance, which occurs all over Bangladesh, causing 25-35\% mortality in chickens and ducks (Chowdhury et al., 1985). It is also known as avian cholera and avian haemorrhagic septicemia and is caused by Pasteurella multocida (Heddleston and Rhoades, 1978). As the BLRI claims that the newly developed chicken, Shuvra is capable to resist diseases and sustain at local environment. So it would be better to check the immune response and protective capability of Shuvra against common infections while dissemination of this newly developed breed of chickens for farming is advocated. So, this piece of research was undertaken to investigate the immune response capability of this strain of birds with Newcastle disease vaccines and Fowl cholera vaccines.

*Corresponding e-mail address: sukumar07@gmail.com

Copyright (C) 2012 Bangladesh Society for Veterinary Medicine

All rights reserved 0238/2012 


\section{MATERIALS AND METHODS}

\section{Newcastle disease vaccine and virus}

Baby Chick Ranikhet Disease Vaccine (BCRDV) and Ranikhet Disease Vaccine (RDV) were obtained from Livestock Research Institute (LRI), Mohakhali, Dhaka. Virulent field isolate of Ranikhet/Newcastle Disease Virus (NDV) used for challenge was obtained from the repository of the Department of Microbiology and Hygiene, BAU, Mymensingh.

\section{Fowl Cholera disease vaccine and bacteria}

FC vaccine called BAU Fowl cholera vaccine was collected from LPVRPC, and used according to the instruction provided by manufacturer. Virulent field isolate of Fowl cholera causing bacteria used for challenge was obtained from the repository of the Department of Microbiology and Hygiene, BAU, Mymensingh.

\section{Experimental chicks}

A total of 40 day-old Shuvra chicks were obtained with the courtesy from BLRI, Savar, Dhaka. The chicks were then reared in the experimental poultry shed and were provided with sufficient nutritional diet and other management requirements with proper biosecurity.

\section{Experimental immunization}

The chicks were divided into four equal groups. Groups A and B were used for NDV vaccines while C and D were used for BAUFC vaccine.

\section{Immunization with NDV and BAUFC vaccines}

Group A were vaccinated primarily with BCRDV through (i/o) route one drop per bird at 7 days of age followed by secondary vaccination with RDV through intramuscular (i/o) route at 35 days $1 \mathrm{~mL}$ per bird. Group B were kept as unvaccinated control. Sera were collected from all the chickens at 7, 21, 35, and 50 days, to determine Haemagglutination Inhibition (HI) antibody titre. Group C were vaccinated primarily with BAUFC vaccine at 42 days of age $(\mathrm{i} / \mathrm{m}) 0.5 \mathrm{~mL}$ per chicken followed by booster vaccination at 70 days of age with the same vaccine, dose and route. Serum was collected from all the chickens at 42, 56, 70, and 84 days to determine PHA antibody titre.

\section{Haemaglutination inhibition (HI) test}

The HI test was performed to determine the HI antibody titres of the sera samples collected from the chickens vaccinated with BCRDV and RDV against NDV. The HI titres of sera samples of control chicken were also analyzed to determine the maternal antibody titres. The test was conducted by using constant 4 HA unit antigen and increasing serum dilution method (Beta-procedure) following the method of Anon (1971). The HI antibody titer of each serum corresponded to reciprocal of highest original dilution of serum inhibiting agglutination of cRBC completely.

\section{Protection test with NDV (Challenge experiment)}

Sixteen days after secondary vaccination with RDV, the chickens of group A and B were challenged with virulent field isolate of NDV intra-nasally $(\mathrm{i} / \mathrm{n}) \quad 0.1 \mathrm{~mL}$ per bird containing $2 \mathrm{ELD}_{50}$ dose, which corresponded to about $100 \%$ mortality in chickens of 10 -weeks of age (Sarkar et al., 2012).

\section{Passive haemagglutination (PHA) test}

The PHA test was performed to determine antibody titres of chickens vaccinated with BAUFC (Group C) and of control (Group D) as per the method described by Tripathy et al. (1970). 


\section{Protection test with FC (Challenge experiment)}

Sixteen days after booster vaccination with BAUFC vaccine the chickens of vaccinated (Group C) and control (Group D) were challenged intramuscularly $(\mathrm{i} / \mathrm{m})$ with virulent field isolate of FC @ $0.5 \mathrm{~mL}$ per bird containing $5.7 \times 10^{9} \mathrm{CFU} / \mathrm{mL}(2.47 \mathrm{OD}$ value at $550 \mathrm{~nm})$ as per the method described by Koly (2011).

\section{Statistical analysis}

Student's t-test was performed to determine significant differences in HI and PHA titres. Protection tests were analyzed by Mantel-Cox log rank test. A P value at $\leq 0.05$ was considered statistically significant.

\section{RESULTS AND DISCUSSION}

\section{HI antibody titres of vaccinated and control chickens}

HI antibody titres in chickens of vaccinated (Group A) and control (Group B) against NDV vaccines at 7, 21, 35, and 50 days of age were converted to $\log 2 \mathrm{HI}$ antibody titres. From this study, it was observed that the HI antibody titres of unvaccinated control (Group B) gradually decreased from day 7 to day 50. The pre-vaccination $\log 2 \mathrm{HI}$ antibody titres of both vaccinated (Group A) and control (Group B) chickens were 6.0 $\pm 0.70,6.00 \pm 0.73$, respectively which gradually declined in control chicken (Group B) to $3.20 \pm 0.44$ on day 50 (Fig. 1). On day 36 antibody titer were $4.00 \pm 0.71$. Rahman et al. (2004) reported a very high titre of maternally derived antibody in 4 day-old chick. Conversely, in vaccinated chicken (Group A), following primary vaccination with BCRDV HI titres were slightly increased at day 21 compared with the control birds (maternal antibody). Similarly, antibody titer of serum that was obtained at day 36 was also slightly ascended compared with day 21 . In day 21,35 and 50 , the HI titre was recorded, $6.20 \pm 0.51,7.10 \pm 0.44,8.8 \pm 70$ respectively (Group A). Similar findings were reported by several investigators (Chowdhury et al., 1981; Kafi et al., 2003; Sarkar et al., 2012).

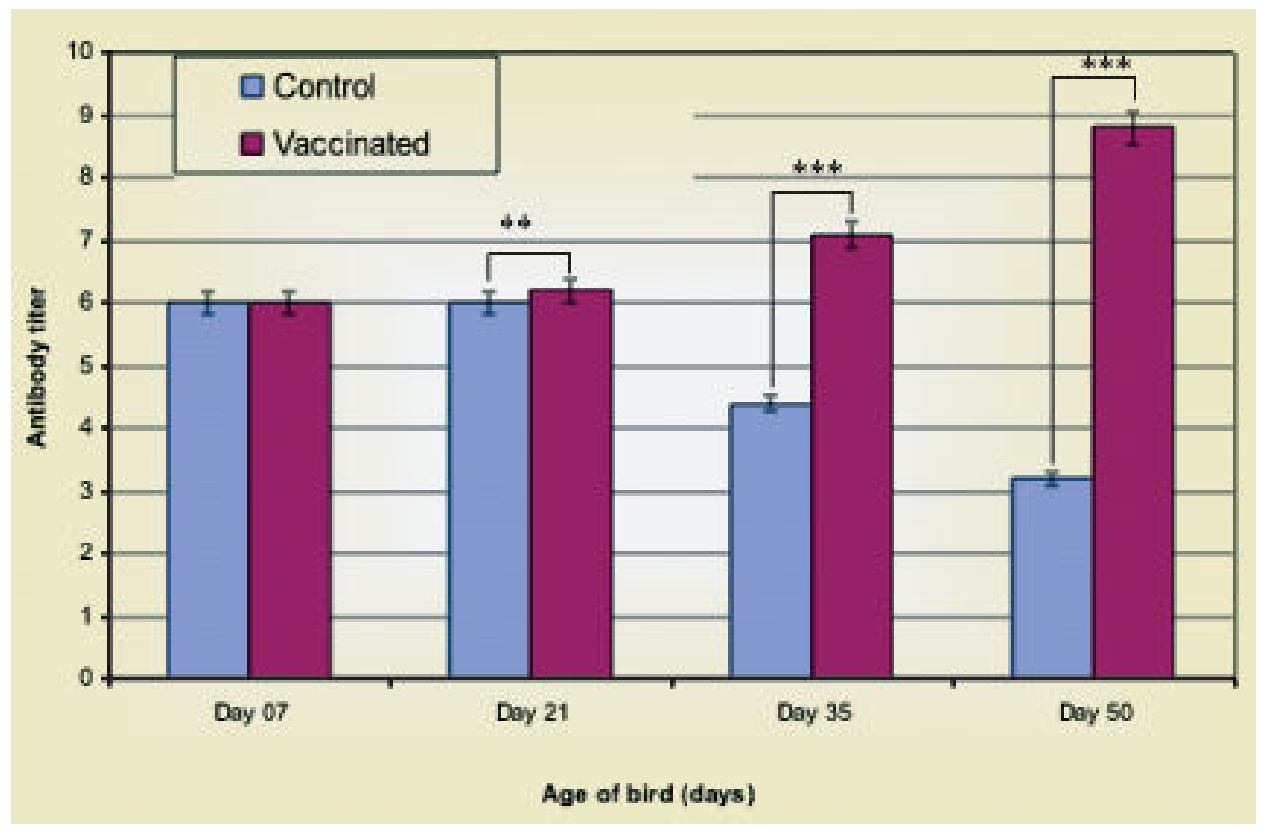

Figure 1. Serum antibody titer ( $\log 2$ base) against NDV antigen in chicken of vaccinated (Group A) and unvaccinated control (Group B). Vaccinated group were immunized with BCRDV through (i/o) route at 7 days of age and with RDV at 35 days of age through $(\mathrm{i} / \mathrm{m})$ route, while control group were not received no vaccine. Blood was drawn at 7, 21, 35, and 50 days of age of birds from both groups. Serum HI antibody titres against NDV antigen were determined by HI test. Results are shown in $\log _{2}$ (Mean $\pm \mathrm{SD}$, where $\mathrm{SD}=\mathrm{Standard}$ deviation, $\mathrm{HI}=$ Haemagglutination inhibition, ${ }^{* *} \mathrm{p}<0.01$ and $\left.* * * \mathrm{p}<0.001\right)$. 


\section{S. Sabrin and others}

\section{Protection test against NDV vaccines}

In case of vaccinated group all the 10 birds exhibited no signs of illness and survived after challenge infection throughout the period of observation of 10 days. Conversely, all the control birds started to show typical clinical signs of ND e.g. sneezing, gasping, greenish-watery diarrhea and drowsiness from $3^{\text {rd }}$ day of challenge infection. Control chickens started to die from $3^{\text {th }}$ day after challenge infection and succumbed within 6 days. The birds also produced typical postmortem lesion for NDV (data not shown). It appeared that birds vaccinated with BCRDV and RDV conferred $100 \%$ protection following virulent challenge (Figure 2).

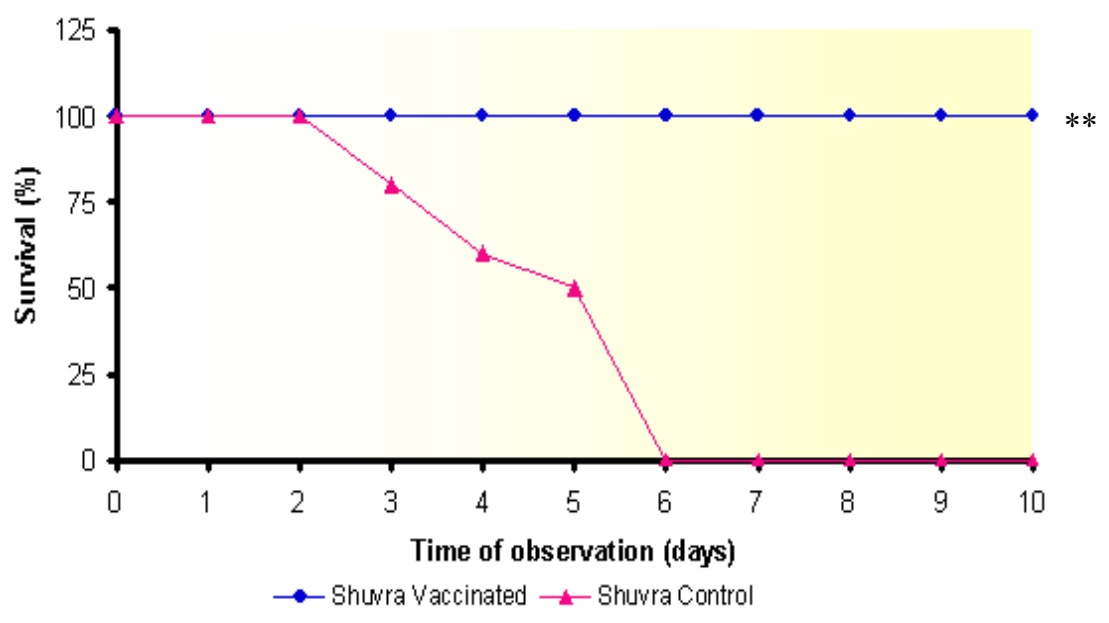

Figure 2. Survival rate of chicken following challenge infection intranasally with virulent field isolate of NDV. The figure show the survival rate of chicken was monitored following intranasal challenge with $2 \mathrm{ELD}_{50}$ dose of NDV and was observed for subsequent 10 days where **p $<0.01$ by Mantal-Cox log rank test.

\section{PHA antibody titres of vaccinated and unvaccinated chickens}

Micro plate PHA test was conducted to determine the antibody titres of test sera collected from the vaccinated (Group C) and unvaccinated control chickens (Group D) used in this experiment and the titres are calculated and presented as mean of titre. Prevaccinated PHA titres of sera samples of all vaccinated and control birds was found to have a mean of $<4.0 \pm 0.00$. The PHA antibody titre of vaccinated chickens was $70.4 \pm 15.67$ after 15 days of primary vaccination and $57.60 \pm 14.31$ after 28 days of primary vaccination (Figure 3 ).

After 15 days of booster vaccination the PHA antibody titre of sera of vaccinated chickens were also determined by microplate PHA test. The antibody titre of sera of Shuvra chickens revealed the mean antibody titre of $179.2 \pm 66.09$. The vaccination dose used for the production of immune response against fowl cholera was closely related with Khan et al. (1997). Booster dose was given with the similar dose and route at 28 days after primary vaccination. Wu et al. (1986) suggested that two doses of FC vaccine were required for better immune response with an interval of two to four weeks after primary vaccination. Similar method to measure the antibody titres following administration of fowl cholera vaccine has been reported by several investigators (Chowdhury et al., 1985; Mondal et al., 1988; Sarker et al., 1992). 




Figure 3. Serum antibody titre against Fowl cholera vaccine in chicken. Chicken was immunized at 6 weeks (42 days) of age while secondary vaccination was done at 10 weeks (70days) of age via I/M route at the dose rate of $0.5 \mathrm{ml}\left(1.75 \times 10^{8} \mathrm{CFU} / \mathrm{ml}\right)$ in each bird. Serum was obtained at $42(6 \mathrm{wks}), 56(8 \mathrm{wks}), 70(10 \mathrm{wks})$ and 84 (12 wks) days of age. Serum antibody titre against vaccination was determined by PHA test. The graph depicts the mean \pm SE values $(\mathrm{n}=5)$. $* * \mathrm{p}<0.01$ by student $\mathrm{t}$-test

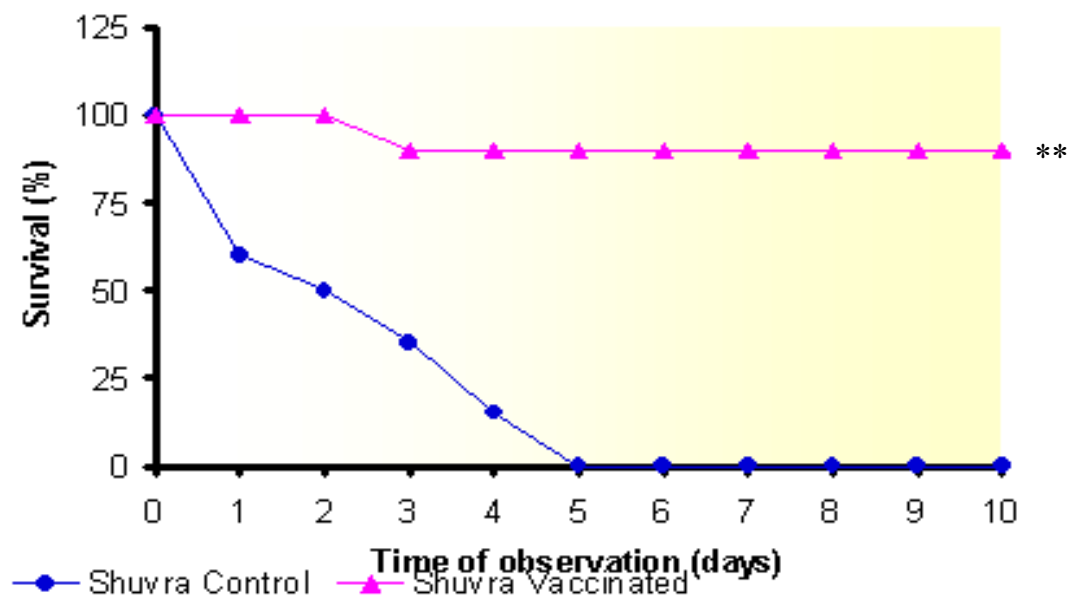

Figure 4. Survival rate of chicken after challenged with virulent $P$. multocida isolate. Chickens were immunized twice with FC vaccine at 6 weeks (42 days) of age and secondly at 10 week (70 days) of age through I/M route at the dose rate of $0.5 \mathrm{ml}\left(1.75 \times 10^{8} \mathrm{CFU} / \mathrm{ml}\right)$. Both vaccinated and control chickens were inoculated with $0.5 \mathrm{ml}$ of fowl cholera culture $(2.47 \mathrm{OD}$ at $550 \mathrm{~nm})$ and the mortality was monitored for subsequent 10 days. $* * \mathrm{p}<0.01$ by Mantel-Cox log rank test. 


\section{Protection test against fowl cholera vaccine}

Sixteen days after final immunization with FC vaccine the chickens were challenged (Figure 4) intramascularly (i/m) with virulent field isolate of FC @ $0.5 \mathrm{ml}$ per bird containing $5.7 \times 10^{9} \mathrm{CFU} / \mathrm{ml}(2.47 \mathrm{OD}$ value at $550 \mathrm{~nm})$.

In post challenge observations, control birds revealed characteristic clinical signs and symptoms of avian pasteurellosis like dullness, depression, anorexia, hyperthermia, laboured breathing, lameness, whitish (chalky) diarrhoea and ultimately death of all birds occurred within 5 days and similar was the observation of Avakian et al. (1989). Vaccinated birds protected themselves and did not show clinical signs except one birds died at $3^{\text {rd }}$ day of observation. Super et al. (2002) observed that the protection rate of the locally isolated P. multocida vaccine was 50-75\% and Avakian et al. (1989) showed a survival rate of $86 \%$. In this experimental study BAU Fowl cholera vaccine protected $90 \%$ challenged Shuvra.

From this investigation, it may be concluded that-

(i) Almost high level of antibody titre was observed in Shuvra following vaccination with either NDV or BAU fowl cholera vaccine.

(ii) Shuvra were able to survive about $90 \%$ in a very hot, humid, congested and crowded environment following challenge.

\section{REFERENCES}

1. Avakian AP, Dick JW, and Derieux WT (1989). Fowl Cholera Immunity Induced by Various Vaccines in Broiler Mini breeder Chickens Determined by Enzyme-Linked Immunosorbent Assay. Avian Diseases 33: 97-102.

2. Awang IPR, Wan-Ahmad-Kusairy WS, and Abdul-Razak J (1992). Detection of maternal antibody against Newcastle disease virus in chicks using an indirect immunoperoxidase test. Journal of Veterinary Malaysia 4(1): 19-23.

3. BBS (Bangladesh Bureau of Statistics) (2009). Report on the Bangladesh Livestock Survey-2008-'09. Govt. of Bangladesh.

4. Chowdhury KA, Amin MM, Rahman A and Ali MR (1985). Investigation of natural outbreak of fowl cholera. Bangladesh Veterinary Journal 19: 49-56

5. FAO, (1999). Report of the FAO World Food Summit Conference, 11. Rome, Italy.

6. Jafari RA, Jalali MR, Ghorbanpoor M, and Saraei SMRM (2008). Effect of dietary garlic on immune response of broiler chicks to live Newcastle disease vaccine. Pakistan Journal Biological Sciences 11(14): $1848-1851$

7. Kafi MA, Rahman MB, Amin MM, Islam MR, Rahman MM and Rahman MK (2003). Comparative serological responses and protection conferred by vaccination with V4HR and BCRDV in chickens. Bangladesh Journal of Veterinary Medicine 1: 25-27.

8. Khan MA, Das PM Choudhury KA, and Islam MR (1997). Pathology of experimentally induced fowl cholera in chickens. Bangladesh Veterinary Journal 31(2): 28-34

9. Koly M. (2011). Evaluation on the efficacy of BAU fowl cholera vaccine. M.S Thesis June-2011. Department of Microbiology and Hygiene, Bangladesh Agricultural University, Mymensingh.

10. MoFL, (2006). National Livestock Policy and Action Plan, Department of Livestock Services, Dhaka.

11. Mondal SK, Rahman MB, Amin MM, Islam MR, Rahman MM, and Sarker AJ (1988). Immune response in chickens induced by Alum precipitated fowl cholera vaccine. I. Humoral immune response. Bangladesh Veterinary Journal 22: 3-4

12. Rahman MB, Rahman MM, Rahman M, Kabir SML, Nazir KHMNH and Amin MM (2004). Efficacy of V4HR Newcastle disease (V4HR-ND) vaccine in broiler birds in Bangladesh. International Journal of Poultry Science 3: 365-368 


\section{S. Sabrin and others}

13. Saleque MA, and Mustafa S (1996). Landless women and poultry. The BRAC model in Bangladesh. Integrated Farming in Human Development. Proceedings of a workshop.

14. Samad MA. (2000). Veterinary practitioner Guide. $1^{\text {st }}$ pub. LEP Pub. No. 07, BAU Campus, Mymensingh.

15. Sarkar SC, Saha S, Amin MM and Hossain MG (2012). The efficacy of Ranikhet disease vaccines produced by Livestock Research Institute of Bangladesh. Microbes and Health 1: 9-13

16. Sarker AJ, Amin MM, and Hossain WMA (1992). Testing and quality control of poultry vaccines and its monitoring in the field. Bangladesh. Agricultural University Research Progress 6: 249-257.

17. Siddique AB, Rahman MB, Amin MM, and Rahman MM (1997). Antibody titres in chicks following pigeon poxvirus inoculation. Bangladesh Veterinarian 14(1-2): 12-14.

18. Super SY, Djaenuri KN, Poerwadikarta B, and Sjafei J (2002). The development of fowl cholera vaccine: 11. Pathogenicity and vaccine protection of $P$. multocida local isolates in experimental ducks. Journal Tmu Terna Dan Veterinar 6(2): 120-125.

19. Tripathy DN, Hanson LE, and Myers WL (1970). Passive haemagglutination test with fowl pox virus. Avian Diseases 14: 29-38.

20. Wu ZJ, Wu LQ, and Cai BX (1986). Comparison between primary and secondary immune responses in chickens vaccinated with fowl cholera attenuated vaccine prepared from P. multocida strain 807. Animal Husbandry and Veterinary Medicine China 18(2): 54-56. 